\title{
ISOSINGULAR LOCI \\ AND THE CARTESIAN PRODUCT STRUCTURE OF COMPLEX ANALYTIC SINGULARITIES
}

BY

\author{
ROBERT EPHRAIM
}

\begin{abstract}
Let $X$ be a (not necessarily reduced) complex analytic space, and let $V$ be a germ of an analytic space. The locus of points $q$ in $X$ at which the germ $X_{q}$ is complex analytically isomorphic to $V$ is studied. If it is nonempty it is shown to be a locally closed submanifold of $X$, and $X$ is locally a Cartesian product along this submanifold. This is used to define what amounts to a coarse partial ordering of singularities. This partial ordering is used to show that there is an essentially unique way to completely decompose an arbitrary reduced singularity as a cartesian product of lower dimensional singularities. This generalizes a result previously known only for irreducible singularities.
\end{abstract}

0. Introduction. Let $X$ be a complex analytic space. For $q \in X, X_{q}$ will denote the germ of $X$ at $q$. In this paper I will study the isosingular loci defined by

Definition 0.1. For $p \in X$ let

$$
\operatorname{Iso}(X, p)=\left\{q \in X \mid X_{q} \cong X_{p}\right\} .
$$

( $\cong$ here and elsewhere will mean complex analytically isomorphic.) It will be shown that:

Theorem 0.2. For any $p \in X, \operatorname{Iso}(X, p)$ is a (possibly 0-dimensional) complex submanifold of some open subset of $X$. Moreover, for any $q \in \operatorname{Iso}(X, p)$ there is an open neighbornood $U$ of $q$, and an analytic space $Y$ such that $U \cong Y \times(U \cap \operatorname{Iso}(X, p))$. ( $\times$ is the cartesian product in the category of analytic spaces.)

This result is used to introduce what is, in effect, a partial ordering of complex analytic singularities in terms of their complexity. This, in turn, is used to study the ways in which a germ of an analytic space may be written as the cartesian product of other germs of analytic spaces. Let $V$ be a germ of an analytic space ( $V$ not the reduced point). By a decomposition of $V$ of length

Received by the editors April 1, 1977.

AMS (MOS) subject classifications (1970). Primary 32C15, 32C40; Secondary 32B10, 32C25.

Key words and phrases. Nonreduced complex space, cartesian product, derivations, complex analytic isomorphism, reduced singularity. 
$k$ I mean an unordered $k$-tuple $\left(V_{1}, \ldots, V_{k}\right)$ of germs of analytic spaces, no $V_{i}$ being the reduced point, such that $V \cong V_{1} \times \cdots \times V_{k}$. (Note that all the $V_{j}$ will be reduced and positive dimensional if and only if $V$ is reduced.) $V$ will be called indecomposable if and only if $V$ has no decomposition of length 2. Finally, $V$ will be called uniquely decomposable if and only if (i) $V$ has a decomposition $\left(V_{1}, \ldots, V_{k}\right)$ with all $V_{j}$ indecomposable, and (ii) if $\left(V_{1}, \ldots, V_{k}\right)$ and $\left(W_{1}, \ldots, W_{h}\right)$ are two such decompositions of $V$, then $k=h$, and, after permuting the $W_{j}^{\prime}$ 's, one has $V_{j} \cong W_{j}$ for all $j$.

It will be shown that:

THEOREM 0.3. If $V$ is a positive dimensional germ of a reduced analytic space, then $V$ is uniquely decomposable.

This generalizes a result from [2]. It would be interesting to know if nonreduced singularities are uniquely decomposable. In particular, this would give a structure theorem for complex analytic Artin local rings.

Finally, let me remark that analogues of these definitions and results can also be formulated for reduced, irreducible germs of real analytic spaces, although the proofs are different [1]. For the purpose of the real analytic analogue of Theorem 0.3, a germ of real analytic space is said to be reduced if the natural map ${ }_{V} \mathbb{Q} \rightarrow{ }_{V} \mathcal{C}$ is injective. $\left({ }_{V} \mathcal{Q}\right.$ here is the real analytic local ring of $V$, and ${ }_{V} \mathcal{C}$ is the ring of germs of continuous functions on $V$.) In particular, if $V$ is a reduced irreducible germ of a complex space, then $V$ is uniquely decomposable both complex analytically and real analytically, and these two decompositions are essentially the same [1]. It is not yet known if this is true for reducible $V$.

I wish to thank the referee for many helpful suggestions, especially for the simple proof he suggested for Lemma 1.5.

1. Preliminaries. Before proceeding with the proof of Theorem 0.2 I collect some useful preliminaries. The bulk of this section is well known, at least for reduced spaces.

I begin by giving the natural generalization of Whitney's first tangent cone [8] to arbitrary germs of analytic spaces. Let $V$ be a germ of analytic space with local ring $v^{\theta}$. Let $\nu^{\mathfrak{m}}$ denote the maximal ideal of $v^{\theta}$ and $e$ : $v^{\theta} \rightarrow \mathbf{C}={ }_{v} \theta /{ }_{v} \mathfrak{m}$ be the natural evaluation map. Recall that one can define the Zariski tangent space of $V, T V=\left\{\mathbf{C}\right.$-derivations $\left.t: V^{\theta} \rightarrow \mathbf{C}\right\}$.

Definition 1.1. $C_{1}(V)=\left\{t \in T V \mid\right.$ there is a C-derivation $\tau:{ }_{\nu} \theta \rightarrow{ }_{v} \theta$ satisfying $t=e \circ \tau\}$.

Clearly $C_{1}(V)$ is a complex linear subspace $T V$ which is a bianalytic invariant of $V$. Let $V$ be embedded as the germ at $0 \in \mathbf{C}^{n}$ of the complex analytic subspace of $\mathbf{C}^{n}$ defined by the ideal $9 \subset{ }_{n} \theta{ }_{n} \theta$ is the ring of germs of holomorphic functions at $\left.0 \in \mathbf{C}^{n}\right)$. Then for $t \in T V$ we have $t \in C_{1}(V)$ if 
and only if there is a germ at $0 \in \mathbf{C}^{n}$ of a holomorphic vector field $U$ such that $U(0)=t$ and $U G \subset 9$.

Now suppose $V$ and $W$ are two germs of analytic spaces. Then, as is well known, we have natural inclusions

$$
T V \subset T(V \times W) \text { and } T W \subset T(V \times W),
$$

such that $T V \cap T W=\{0\}$ and $T(V \times W)=T V \oplus T W$. Moreover, we have

Lemma 1.2. $C_{1}(V \times W)=C_{1}(V) \oplus C_{1}(W)$.

PRoof. The proof is easy and is left to the reader.

$C_{1}(V)$ is interesting because

LEMMA 1.3. Let $V$ be the germ at $0 \in \mathbf{C}^{n}$ of the analytic subspace of $\mathbf{C}^{n}$ defined by the ideal $G \subset{ }_{n} \mathcal{O}$. Then there is a germ of a holomorphic vector field

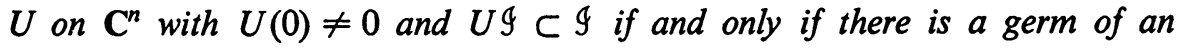
analytic space $W$ such that $V \cong W \times \mathbf{C}_{0}$. $\left(\mathbf{C}_{0}\right.$ denotes the germ of $\mathbf{C}$ at $0 \in \mathbf{C}$.)

Proof. See [3, §2.12].

COROLlaRY 1.4. Let $V$ be the germ at $0 \in \mathbf{C}^{n}$ of the analytic subspace of $\mathbf{C}^{n}$ defined by the ideal $G \subset{ }_{n} \theta$. Then there are $k$ germs of holomorphic vector fields $U_{1}, \ldots, U_{k}$ which preserve $G$ and such that $U_{1}(0), \ldots, U_{k}(0)$ are linearly independent if and only if there is a germ of an analytic space $W$ such that $V \cong W \times \mathbf{C}_{0}^{k}$. Also, $d=\operatorname{dim}_{\mathbf{C}} C_{1}(V)$ is the greatest such $k$.

PROof. The corollary follows from repeated applications of Lemma 1.3, the repeated applications being justified by Lemma 1.2.

I finish the preliminaries with

LEMMA 1.5. If $V$ and $W$ are germs of analytic spaces such that $V \times \mathbf{C}_{0} \cong$ $W \times \mathbf{C}_{0}$, then $V \cong W$.

Proof. The proof is based on an elementary remark. Let $Z \subset \mathbf{C}_{0}^{n}$ be any germ of an analytic space. Then $Z \times \mathbf{C}_{0}^{k} \subset \mathbf{C}_{0}^{n+k}$ in a natural way, and clearly $Z \cong\left(Z \times \mathbf{C}_{0}^{k}\right) \cap\left(\mathbf{C}_{0}^{n} \times\{0\}\right)$. But more is true. If $M \subset \mathbf{C}_{0}^{n+k}$ is any germ of a complex $n$-manifold transverse to $\{0\} \times \mathbf{C}_{0}^{k}$, then $Z \cong M \cap(Z \times$ $\left.\mathbf{C}_{0}^{k}\right)$. To see this choose coordinates $\left(x_{1}, \ldots, x_{n}\right)$ on $\mathbf{C}_{0}^{n}$ and coordinates $\left(y_{1}, \ldots, y_{k}\right)$ on $\mathbf{C}_{0}^{k}$. Then $M$ will be defined by equations $y_{j}-f_{j}\left(x_{1}, \ldots, x_{n}\right)$ $=0, j=1, \ldots, k$. The mapping which sends $\left(x_{1}, \ldots, x_{n}, y_{1}, \ldots, y_{k}\right)$ to $\left(x_{1}, \ldots, x_{n}, y_{1}-f_{1}\left(x_{1}, \ldots, x_{n}\right), \ldots, y_{k}-f_{k}\left(x_{1}, \ldots, x_{n}\right)\right)$ is an isomorphism of $\mathbf{C}_{0}^{n+k}$ to itself which gives, by restriction, an explicit isomorphism

$$
M \cap\left(Z \times \mathbf{C}_{0}^{k}\right) \cong\left(\mathbf{C}_{0}^{n} \times\{0\}\right) \cap\left(Z \times \mathbf{C}_{0}^{k}\right) \cong Z .
$$

Proof of Lemad 1.5. By Lemma 1.2, $\operatorname{dim} C_{1}(V)=\operatorname{dim} C_{1}(W)=k-1$. 
So, by Corollary 1.4, $V \cong V^{\prime} \times \mathrm{C}_{0}^{k-1}$ and $W=W^{\prime} \times \mathrm{C}_{0}^{k-1}$ for some $V^{\prime}$ and $W^{\prime}$ with $\operatorname{dim} C_{1}\left(V^{\prime}\right)=\operatorname{dim} C_{1}\left(W^{\prime}\right)=0$. To prove $V \cong W$ it suffices to show $V^{\prime} \cong W^{\prime}$. By assumption $V^{\prime} \times \mathrm{C}_{0}^{k} \cong W^{\prime} \times \mathrm{C}_{0}^{k}$.

Suppose $V^{\prime}$ and $W^{\prime}$ are embedded as germs in $\mathrm{C}_{0}^{n}$. Then any isomorphism $\Omega: V^{\prime} \times C_{0}^{k} \rightarrow W^{\prime} \times C_{0}^{k}$ extends to an isomorphism $\Omega: \mathbf{C}_{0}^{n+k} \rightarrow \mathbf{C}_{0}^{n+k}$. Let $M \subset \mathbf{C}_{0}^{n+k}$ be a germ of a complex $n$-manifold transverse to $\{0\} \times \mathbf{C}_{0}^{k}$. Then by the above remark,

$$
V^{\prime} \cong M \cap\left(V^{\prime} \times \mathbf{C}_{0}^{k}\right) \cong \Omega(M) \cap \Omega\left(V^{\prime} \times \mathbf{C}_{0}^{k}\right) \cong \Omega(M) \cap\left(W^{\prime} \times \mathbf{C}_{0}^{k}\right),
$$

and it suffices to show that $\Omega(M)$ is transverse to $\{0\} \times \mathbf{C}_{0}^{k}$.

By construction

$$
C_{1}\left(V^{\prime} \times \mathbf{C}_{0}^{k}\right)=C_{1}\left(W^{\prime} \times \mathbf{C}_{0}^{k}\right)=T\left(\{0\} \times \mathbf{C}_{0}^{k}\right) .
$$

The choice of $M$ gives

$$
T M \cap C_{1}\left(V^{\prime} \times \mathbf{C}_{0}^{k}\right)=T M \cap T\left(\{0\} \times \mathbf{C}_{0}^{k}\right)=\{0\} .
$$

Thus

$$
\begin{aligned}
T \Omega(M) \cap T\left(\{0\} \times \mathbf{C}_{0}^{k}\right) & =T \Omega(M) \cap C_{1}\left(W^{\prime} \times \mathbf{C}_{0}^{k}\right) \\
& =T \Omega(M) \cap C_{1}\left(\Omega\left(V^{\prime} \times C_{0}^{k}\right)\right)=\{0\}
\end{aligned}
$$

and we are done.

2. Proof of Theorem 0.2. I now turn my attention to Theorem 0.2. Let $X$ be an analytic space and $p \in X$. Then clearly, for $q \in \operatorname{Iso}(X, p)$ one has $\operatorname{Iso}(X, q)=\operatorname{Iso}(X, p)$, so that Theorem 0.2 is purely local and may be restated as

THEOREM 2.1. Let $X$ be an analytic space and let $p \in X$. Then there is an open neighborhood $U$ of $p$ and an analytic space $Y$ such that $\operatorname{Iso}(X, p) \cap U$ is a (possibly 0-dimensional) complex submanifold of $U$ and

$$
U \cong Y \times(\operatorname{Iso}(X, p) \cap U) .
$$

Proof. The proof of this theorem will take the rest of this section. It is convenient to begin with a definition.

Definition 2.2. For $p \in X$ let $M(X, p)$ be the smallest germ at $p$ of an analytic subspace of $X$ such that $\operatorname{Iso}(X, p)_{p} \subset M(X, p)$. (Iso $(X, p)_{p}$ denotes the germ at $p$ of $\operatorname{Iso}(X, p)$.)

$M(X, p)$ certainly exists because the local ring ${ }_{x} \theta_{p}$ of $X_{p}$ is noetherian. Moreover, $M(X, p)$ is a reduced germ because of its minimality. Also, if $\psi$ : $X_{p} \rightarrow X_{q}$ is an isomorphism, then $\psi$ induces an isomorphism $\psi: \operatorname{Iso}(X, p)_{p} \rightarrow$ $\operatorname{Iso}(X, p)_{q}=\operatorname{Iso}(X, q)_{q}$, so that $\psi$ must also induce an isomorphism $\psi$ : $M(X, p) \rightarrow M(X, q)$.

LEMma 2.3. $M(X, p)$ is a (possibly 0-dimensional) germ of a submanifold of $X_{p}$. 
Proof. Choose a neighborhood $U$ of $p$ small enough to find an analytic subspace $M$ of $U$ satisfying $M_{p}=M(X, p)$. By shrinking $U$ we may assume that $\operatorname{Iso}(X, p) \cap U \subset M$, and also that $\operatorname{dim} M_{q}<\operatorname{dim} M(X, p)$ for all $q \in$ $M$.

By the minimality of $M(X, p)$ we have $\operatorname{Iso}(X, p)_{p} \not \subset \operatorname{Sg}(M(X, p))=$ $(\operatorname{Sg}(M))_{p}$. Hence there is a $q \in \operatorname{Iso}(X, p) \cap U \subset M$ for which $M_{q}$ is the germ of a manifold. Since $q \in \operatorname{Iso}(X, p)$ we have $\operatorname{Iso}(X, q)_{q}=\operatorname{Iso}(X, p)_{q} \subset M_{q}$ so that $M(X, q) \subset M_{q}$ and $\operatorname{dim} M(X, q) \leqslant \operatorname{dim} M_{q}$. But $q \in \operatorname{Iso}(X, p)$ also gives $M(X, q) \cong M(X, p)$ so that $\operatorname{dim} M(X, q)=\operatorname{dim} M(X, p) \geqslant \operatorname{dim} M_{q}$. Thus, in fact, $\operatorname{dim} M(X, q)=\operatorname{dim} M_{q}$. This, together with $M(X, q) \subset M_{q}$ and the fact that $M_{q}$ is an irreducible germ, gives $M(X, q)=M_{q}$, which is a germ of a manifold. But $M(X, p) \cong M(X, q)$ and the lemma is proven.

Note that $\operatorname{dim} M(X, p)=0$ if and only if $p$ is an isolated point of Iso $(X, p)$, and in this case Theorem 2.1 is trivial. For the rest of this section I will assume $\operatorname{dim} M(X, p)=n \geqslant 1$.

REMARK 2.4. Since Theorem 2.1 is purely local in a neighborhood of $p$, we may shrink $X$ by replacing $X$ with a small open neighborhood of $p \in X$. This allows us to put $X$ in a convenient form.

In this way we may suppose we have a connected submanifold $M \subset X$ such that $\operatorname{Iso}(X, p) \subset M$, and $M(X, p)=M_{p}$. Then, for all $q \in \operatorname{Iso}(X, p)$ we have $\operatorname{Iso}(X, q)=\operatorname{Iso}(X, p) \subset M$, and thus $M(X, q) \subset M_{q}$. But for $q \in$ Iso $(X, p)$ we have $M(X, q) \cong M(X, p)=M_{p} \cong M_{q}$, and we get $M(X, q)=$ $M_{q}$ for all $q \in \operatorname{Iso}(X, p)$.

We may also assume that $X$ is embedded as an analytic subspace of a polydisc $\Delta, 0 \in \Delta \subset \mathbf{C}^{n+m}$ (where $(x, y)=\left(x_{1}, \ldots, x_{n}, y_{1}, \ldots, y_{m}\right)$ give the coordinates on $\left.\mathbf{C}^{n+m}\right)$, and that the embedding is such that $p=0 \in \mathbf{C}^{n+m}$ and $M=\Delta \cap\left(C^{n} \times\{0\}\right)=\Delta \cap\left\{\left(x_{1}, \ldots, x_{n}, 0, \ldots, 0\right)\right\}$. Finally, we may also assume that we have holomorphic functions $f_{1}, \ldots, f_{r}$ on $\Delta$ which globally generate the coherent ideal sheaf defining $X$ in $\Delta$, and whose germs at $0 \in \mathbf{C}^{n+m}, f_{10}, \ldots, f_{r 0}$, give a minimal set of generators for the defining ideal of the germ $X_{0}$. This setup will be fixed for the rest of this section.

OBSERVATION 2.5. Theorem 2.1 will follow if it can be shown that $\operatorname{dim} C_{1}\left(X_{0}\right)>n=\operatorname{dim} M(X, 0)$.

Proof. It would then follow from Corollary 1.4 that there is an analytic space $Y$, a domain $D \subset \mathbf{C}^{\prime}\left(t=\operatorname{dim} C_{1}\left(X_{0}\right)\right)$, a neighborhood $U$ of 0 in $X$, and an isomorphism $\psi: Y \times D \rightarrow U$. Let $\left(y_{0}, d_{0}\right)=\psi^{-1}(0) \in Y \times D$. Then $\left\{y_{0}\right\} \times D \subset \operatorname{Iso}\left(Y \times D, \psi^{-1}(0)\right)$ so that $\psi\left(\left\{y_{0}\right\} \times D\right) \subset \operatorname{Iso}(X, 0) \cap U \subset$ $M \cap U$. Since $\psi\left(\left\{y_{0}\right\} \times D\right)$ and $M \cap U$ are submanifolds of $U$, and $\operatorname{dim} \psi\left(\left\{y_{0}\right\} \times D\right)=t \geqslant n=\operatorname{dim}(M \cap U)$, it follows that $t=n$ and $\psi\left(\left\{y_{0}\right\}\right.$ $\times D)$ is just the union of components of $M \cap U$. Shrinking $Y, D$, and $U$ we can achieve $\psi\left(\left\{y_{0}\right\} \times D\right)=M \cap U$. But then $\operatorname{Iso}(X, 0) \cap U=M \cap U$, a 
submanifold of $U$, and the result follows by using the isomorphism $\psi$ : $\left\{y_{0}\right\} \times D \rightarrow M \cap U=\operatorname{Iso}(X, 0) \cap U$ to identify $D$ and $\operatorname{Iso}(X, 0) \cap U$.

I now give a construction of Seidenberg [5], [6] which will be used to show $\operatorname{dim} C_{1}\left(X_{0}\right) \geqslant n$. Intuitively, the construction gives, for any natural number $k$, an algebraic variety whose points are certain $k$-jets of $k$-equivalences of $X_{0}$, and a constructible set whose points are certain " $k$-jets" of germs $V k$-equivalent to $X_{0}$. Recall that the germs $V$ and $W$ are $k$-equivalent if ${ }_{V} \theta /{ }_{V} \mathrm{~m}^{k+1}$ $\cong{ }_{W} \theta /{ }_{W} \mathfrak{m}^{k+1}\left({ }_{\nu} \mathfrak{m}\right.$ and ${ }_{W} \mathfrak{m}$ are the maximal ideals in ${ }_{\nu} \theta$ and $\left.{ }_{W} \theta\right)$.

Let $g_{1}(P, x, y), \ldots, g_{r}(P, x, y)$ be polynomials of degree $k$ in the variables $(x, y)$ with indeterminant coefficients which I collectively denote by $(P)$ (just as $(x)$ collectively denotes $\left.\left(x_{1}, \ldots, x_{n}\right)\right)$. Let $a_{i j}(Q, x, y), 1 \leqslant i, j \leqslant r$, be polynomials of degree $k$ in the $(x, y)$ with indeterminant coefficients which I collectively denote by $(Q)$. Let $\varphi_{1}(R, x, y), \ldots, \varphi_{n}(R, x, y)$, $\psi_{1}(R, x, y), \ldots, \psi_{m}(R, x, y)$ be polynomials of degree $k$ in $(x, y)$ such that $\varphi_{i}(R, 0,0)=0,1 \leqslant i \leqslant n$, and $\psi_{j}(R, x, 0)=0,1 \leqslant j \leqslant m$, and having indeterminant coefficients which I collectively denote by $(R)$. For convenience I let $\operatorname{Jac}(\varphi, \psi)(0)$ denote the jacobian of $\left(\varphi_{1}, \ldots, \varphi_{n}, \psi_{1}, \ldots, \psi_{m}\right)$ with respect to the $(x, y)$ evaluated at $(x, y)=(0,0) \cdot \operatorname{Jac}(\varphi, \psi)(0)$ is a polynomial in the $(R)$ 's. Finally let $S$ be an indeterminant.

The $(P)$ give coordinates on some affine space $\mathrm{C}^{M(k)}$. The $(P, Q, R, S)$ give coordinates on some affine space $\mathbf{C}^{N(k)}$. Let $\pi_{P}: \mathbf{C}^{N(k)} \rightarrow \mathbf{C}^{M(k)}$ be defined by $\pi_{P}(P, Q, R, S)=(P)$.

Let $T_{0} f_{1}, \ldots, T_{0} f_{r}$ be the Taylor expansions about $0 \in \mathbf{C}^{n+m}$ of $f_{1}, \ldots, f_{r}$ (which are chosen as in Remark 2.4). Consider the conditions:

$$
g_{i}(P, \varphi(R, x, y), \psi(R, x, y))-\sum a_{i j}(Q, x, y) T_{0} f_{j}(x, y)
$$

are in the $(k+1)$ st power of the ideal generated by the

$$
\left(x_{1}, \ldots, x_{n}, y_{1}, \ldots, y_{m}\right) \text { for } 1 \leqslant i \leqslant r \text {. }
$$

These conditions are equivalent to a finite number of polynomial equations in the $(P, Q, R)$. These equations, together with the polynomial equation

$$
S \cdot \operatorname{Jac}(\varphi, \psi)(0) \cdot \operatorname{det}\left\|a_{i j}(Q, 0,0)\right\|=1,
$$

define an analytic subspace of $\mathbf{C}^{N(k)}$. Let $A(k)$ be the reduction of this analytic space. Then $A(k)$ is a finite union of affine subvarieties of $\mathbf{C}^{N(k)}$. Let $B(k)=\pi_{P}(A(k))$. Then $B(k)$ is a constructible subset of $C^{M(k)}[4$, p. 97].

Now, in $\mathbf{C}^{n}$ having coordinates $(z)=\left(z_{1}, \ldots, z_{n}\right)$ define a polydisc $\Delta^{\prime} \subset \mathbf{C}^{n}$ by $\Delta^{\prime}=\left\{(z) \in \mathbf{C}^{n} \mid(z, 0) \in \Delta \subset \mathbf{C}^{n+m}\right\}$. (Here $\Delta$ is as defined in Remark 2.4.) For a fixed $(z) \in \Delta^{\prime}$ and for a function $h$ holomorphic on a neighborhood of $(z, 0)$ in $\Delta, h(z+x, y)$ is a function holomorphic near $0 \in \mathbf{C}^{n+m}$. I let $T_{z} h$ denote the Taylor expansion in the variables $(x, y)$ centered at $(x, y)=(0,0)$ of the function $h(z+x, y)$. I will let $T_{z}^{k} h$ denote the polynomial one gets 
from $T_{z} h$ by discarding all terms of order greater than $k$. Note that the coefficients of $T_{z} h$ are just the values at $(z, 0)$ of various derivatives of $h$. Thus, these coefficients vary holomorphically with $(z)$.

Let $f_{1}, \ldots, f_{r}$ be as in Remark 2.4. Then the coefficients of $T_{z}^{k} f_{1}, \ldots, T_{z}^{k} f_{r}$ define a holomorphic map $T(k): \Delta \rightarrow \mathbf{C}^{M(k)}$.

REMARK 2.8. If $(z) \in T(k)^{-1}(B(k))$, then $X_{(z, 0)}$ is $k$-equivalent to $X_{0}$. In fact, any point of $A(k) \cap \pi_{P}^{-1}(T(k)(z))$ gives a germ of an isomorphism $(\varphi(k), \psi(k)): \mathbf{C}_{0}^{n+m} \rightarrow \mathbf{C}_{0}^{n+m}$ (defined by polynomials) and an $r \times r$ matrix $\left\|a_{i j}(k)\right\| \in \mathrm{Gl}\left(r,{ }_{n+m} \mathcal{O}\right)$ (defined by polynomials) which satisfy

$$
T_{z}^{k} f_{i}(\varphi(k), \psi(k))-\sum a_{i j}(k) T_{0} f_{j} \in_{n+m} \mathfrak{m}^{k+1}, \quad 1 \leqslant i \leqslant r .
$$

This is equivalent to

$$
\begin{aligned}
& f_{i}(z+\varphi(k)(x, y), \psi(k)(x, y))-\sum a_{i j}(k)(x, y) f_{j}(x, y) \\
& \in_{n+m} \mathrm{~m}^{k+1}, \quad 1 \leqslant i \leqslant r .
\end{aligned}
$$

This shows that $(z+\varphi(k), \psi(k)): \mathbf{C}_{0}^{n+m} \rightarrow \mathbf{C}_{(z, 0)}^{n+m}$ defines $k$-equivalence from $X_{0}$ to $X_{(z, 0)}$.

REMARK 2.11. If $z \in T(k)^{-1}(B(k))$ for all $k$, then $X_{0} \cong X_{(z, 0)}$. Thus $(z, 0)$ $\in \operatorname{Iso}(X, 0)$.

Proof. By Remark 2.8, for each $k$ we have a germ of an isomorphism $(\varphi(k), \psi(k)): \mathbf{C}_{0}^{n+m} \rightarrow \mathbf{C}_{0}^{n+m}$ and an $\left\|a_{i j}(k)\right\| \in \mathrm{Gl}\left(r,{ }_{n+m}()\right)$ satisfying (2.10). I apply Wavrik's [7] extension of Artin's theorem on solutions of analytic equations. By this result, for $k$ sufficiently large we can find a germ of a map $(\varphi, \psi): \mathbf{C}_{0}^{n+m} \rightarrow \mathbf{C}_{0}^{n+m}$ and $a_{i j} \in_{n+m} \mathcal{O}, 1 \leqslant i, j \leqslant r$, which satisfy

(a) $\varphi_{i}-\varphi_{i}(k) \in_{n+m} \mathfrak{m}^{2}, \quad 1 \leqslant i \leqslant n$,

(b) $\psi_{j}-\psi_{j}(k) \in_{n+m} \mathrm{~m}^{2}, \quad 1 \leqslant j \leqslant m$,

(c) $a_{i j}-a_{i j}(k) \in_{n+m} \mathrm{~m}^{2}, \quad 1 \leqslant i, j \leqslant r$ and

(d) $f_{i}(z+\varphi(x, y), \psi(x, y))-\sum a_{i j}(x, y) f_{j}(x, y)=0$,

$$
1 \leqslant i \leqslant r .
$$

(2.12)(a) and (b) show that $(\varphi, \psi): \mathbf{C}_{0}^{n+m} \rightarrow \mathbf{C}_{0}^{n+m}$ is a germ of an isomorphism and (2.12)(c) shows that $\left\|a_{i j}\right\| \in \mathrm{Gl}\left(r,{ }_{n+m} \theta\right)$. With these facts in mind, (2.12)(d) shows that $(z+\varphi, \psi): \mathbf{C}_{0}^{n+m} \rightarrow \mathbf{C}_{(z, 0)}^{n+m}$ induces an isomorphism $X_{0} \rightarrow$ $X_{(z, 0)}$.

REMARK 2.13. If $(z, 0) \in \operatorname{Iso}(X, 0)$ then $z \in T(k)^{-1}(B(k))$ for all $k$.

I now finish the proof of Theorem 2.1 with

Proposition 2.15. $\operatorname{dim} C_{1}\left(X_{0}\right)=\operatorname{dim} M(X, 0)=n$.

Proof. The argument of Observation 2.5 shows that $\operatorname{dim} C_{1}\left(X_{0}\right) \leqslant n$. The other inequality must be proven. 
Since $B(k)$ is constructible we can write $T(k)^{-1}(B(k)) \times\{0\} \subset M$ as a finite union $T(k)^{-1}(B(k)) \times\{0\}=\cup\left(F_{i}-G_{i}\right)$ where each $F_{i}$ and $G_{i}$ are analytic subsets of $M$, and $G_{i}$ contains no irreducible component of $F_{i}$. By Remark 2.13 we have Iso $(X, 0) \subset T(k)^{-1}(B(k)) \times\{0\} \subset \cup F_{i} \subset M$. But then Iso $(X, 0)_{0} \subset \cup F_{i 0} \subset M_{0}=M(X, 0)$. By the minimality of $M(X, 0)$ it follows that $\cup F_{i 0}=M_{0}$. But, since $M_{0}$ is irreducible, there is an $i$, say $i=1$, so that $F_{10}=M_{0}$. But then $F_{1}=M, G_{1}$ is a proper analytic subset of $M$, and $M-G_{1} \subset T(k)^{-1}(B(k)) \times\{0\}$.

$G_{1}$, of course, depends on $k$. Putting this dependency into the notation, write $G(k)=G_{1}$. Let $H$ denote the union of all the $G(k)$. Then $M-H$ is dense in $M$ and $M-H \subset T(k)^{-1}(B(k)) \times\{0\}$ for every $k$. Thus, by Remark 2.11, we have $M-H \subset \operatorname{Iso}(X, 0)$. Thus, Iso $(X, 0)$ is dense in $M$.

Now for a positive integer $k$ define $E(k) \subset \Delta^{\prime} \times C^{N(k)}$ by

$$
E(k)=\{(z, P, Q, R, S) \mid(P, Q, R, S) \in A(k) \text { and } T(k)(z)=P\} .
$$

$E(k)$ carries in a natural way the structure of a reduced analytic space, and I will suppose it is so endowed. Let $\pi_{1}: E(k) \rightarrow \Delta^{\prime}$ be defined by $\pi_{1}(z, P, Q, R, S)=(z) . E(k)$ is constructed so that $\pi_{1}(E(k))=$ $T(k)^{-1}(B(k))$. But then $M-G(k) \subset \pi_{1}(E(k)) \times\{0\}$ so that $\pi_{1}(E(k))$ contains a dense open subset of $\Delta^{\prime}$. Since $E(k)$ is second countable, it follows that $D(k)=$ \{regular points of $E(k)$ at which $\operatorname{rank}\left(\left.\pi_{1}\right|_{E(k)}\right)=n$ \} is a nonempty open subset of $E(k)$ [9, Chapter 4, Theorem $8 D] . \pi_{1}(D(k))$ is an open (in fact a dense open) subset of $\Delta^{\prime}$. Note that for any $(w) \in \pi_{1}(D(k))$ we can find a section of $\left.\pi_{1}\right|_{E(k)}$ on a neighborhood of $(w)$ (by the implicit function theorem).

Since Iso $(X, 0)$ is dense in $M$, we can find a $(w) \in \pi_{1}(D(k))$ such that $(w, 0) \in \operatorname{Iso}(X, 0)$. Choose a section of $\left.\pi_{1}\right|_{E(k)}$ over a neighborhod $U$ of $(w)$. This section gives holomorphic functions on $U \times \mathbf{C}^{n+m}, a_{i j}(k)(z, x, y), 1 \leqslant i$, $j<r ; \varphi_{i}(k)(z, x, y), 1<i<n$; and $\psi_{j}(k)(z, x, y), 1<j<m$. All these are polynomials in $(x, y)$ of degree $k$ with coefficients being holomorphic functions on $U$. Moreover,

(2.16)(a) det $\left\|a_{i j}(k)(z, 0,0)\right\|$ is a nonvanishing holomorphic function on $U$.

(b) $\varphi_{i}(k)(z, 0,0)=0,1 \leqslant i \leqslant n ; \psi_{j}(k)(z, x, 0)=0,1 \leqslant j \leqslant m$. And for each fixed $z \in U,(\varphi(k), \psi(k))=\left(\varphi_{1}(k), \ldots, \varphi_{n}(k), \psi_{1}(k), \ldots, \psi_{m}(k)\right)$ defines a germ of an isomorphism $\mathbf{C}_{0}^{n+m} \rightarrow \mathbf{C}_{0}^{n+m}$.

(c) For each $i, 1 \leqslant i<r$,

$$
f_{i}(z+\varphi(k)(z, x, y), \psi(k)(z, x, y))-\sum a_{i j}(k)(z, x, y) f_{j}(x, y)
$$

is in the $(k+1)$ st power of the ideal generated by the $(x, y)$.

I want to transfer this information from $(w) \in \Delta^{\prime}$ to $(0) \in \Delta^{\prime}$. This can be done because $(w, 0) \in \operatorname{Iso}(X, 0)$. We have $\left\|a_{i j}\right\| \in \mathrm{Gl}\left(r,{ }_{n+m} \theta\right)$ and a germ of an isomorphism $(\varphi, \psi): \mathrm{C}_{0}^{n+m} \rightarrow C_{0}^{n+m}$ such that 


$$
f_{i}(w+\varphi(x, y), \psi(x, y))=\sum a_{i j}(x, y) f_{j}(x, y), \quad 1<i<r,
$$

and by Remark 2.4 we automatically have $\psi_{1}(x, 0)=\cdots=\psi_{m}(x, 0)=0$.

Let $\left\|b_{i j}\right\| \in \mathrm{Gl}\left(r,{ }_{n+m} \Theta\right)$ be defined by the condition $\left\|b_{i j}(\varphi(x, y), \psi(x, y))\right\|$ $=\left\|a_{i j}(x, y)\right\|^{-1}$. Let

$$
(\lambda, \tau)=\left(\lambda_{1}, \ldots, \lambda_{n}, \tau_{1}, \ldots, \tau_{m}\right): \mathbf{C}_{0}^{n+m} \rightarrow \mathbf{C}_{0}^{n+m}
$$

be the inverse of $(\varphi, \psi): \mathbf{C}_{0}^{n+m} \rightarrow \mathbf{C}_{0}^{n+m}$. Since $\psi(x, 0)=0$, it follows that $\tau(x, 0)=0$ and also that $\lambda(\varphi(x, 0), 0)=(x)$. For convenience I write $\mu(x)=$ $\varphi(x, 0)$, so that $\lambda(\mu(x), 0)=(x)$. Equations (2.17) are equivalent to

$$
f_{i}(\lambda(x, y), \tau(x, y))=\sum b_{i j}(x, y) f_{j}(w+x, y), \quad 1 \leqslant i<r .
$$

Let $(t)=\left(t_{1}, \ldots, t_{n}\right)$, so that $(t, x, y)$ give coordinates on $\mathbf{C}^{2 n+m}$. Then $\varphi_{i}(k)(w+\mu(t), x, y), 1 \leqslant i<n$, and $\psi_{j}(k)(w+\mu(t), x, y), 1<j<m$, define germs in $2 n+m$, and $\left\|a_{i j}(k)(w+\mu(t), x, y)\right\| \in \mathrm{Gl}(r, 2 n+m \mathcal{\theta})$.

Define $\left\|\alpha_{i j}(k)(t, x, y)\right\| \in \mathrm{Gl}(r, 2 n+m \theta)$ to be the product of the matrices

$$
\left\|b_{i l}(\mu(t)+\varphi(k)(w+\mu(t), x, y), \dot{\psi}(k)(w+\mu(t), x, y))\right\|
$$

on the left and $\left\|a_{j j}(k)(w+\mu(t), x, y)\right\|$ on the right. Define

$$
\omega(k)(t, x, y)=\lambda(\mu(t)+\varphi(k)(w+\mu(t), x, y), \psi(k)(w+\mu(t), x, y))-(t),
$$

and define

$$
\gamma(k)(t, x, y)=\tau(\mu(t)+\varphi(k)(w+\mu(t), x, y), \psi(k)(w+\mu(t), x, y)) .
$$

Now, using the equations $\lambda(0,0)=\tau(x, 0)=0, \lambda(\mu(x), 0)=(x),(2.16)(b)$, and the fact that a composite of isomorphisms is an isomorphism we get

$$
\begin{aligned}
& \omega(k)(t, 0,0)=0, \gamma(k)(t, x, 0)=0 \text { and, the map } \\
& (\omega(k)(0, x, y), \gamma(k)(0, x, y)): \mathbf{C}_{0}^{n+m} \rightarrow \mathbf{C}_{0}^{n+m} \text { is a germ of an } \\
& \text { isomorphism. }
\end{aligned}
$$

Now, replacing $(x)$ in (2.18) by $\mu(t)+\varphi(k)(w+\mu(t), x, y)$ and $(y)$ by $\psi(k)(w+\mu(t), x, y)$, and using (2.16)(c) with (z) replaced by $(w+\mu(t))$, we get

$$
\begin{aligned}
f_{i}(t+\omega(k)(t, x, y), \gamma(k)(t, x, y))- & \sum \alpha_{i j}(k)(t, x, y) f_{j}(x, y) \\
& \in_{2 n+m} \mathfrak{m}^{k+1} \quad \text { for } 1 \leqslant i \leqslant r .
\end{aligned}
$$

This completes the transfer of the information of $(2.16)$ from $(w) \in \Delta^{\prime}$ to $0 \in \Delta^{\prime}$.

Now, since we have the $\omega(k), \gamma(k)$, and $\left\|\alpha_{i j}(k)\right\|$ satisfying (2.20) for every $k$, we can again apply Wavrik [7]. Then, choosing a sufficiently large $k$, we get $\alpha_{i j} \in{ }_{2 n+m} \mathcal{\theta}, 1 \leqslant i, j \leqslant r, \omega_{i} \in{ }_{2 n+m} \mathcal{O}, 1 \leqslant i \leqslant n$, and $\gamma_{j} \in{ }_{2 n+m} \mathcal{\theta}$, $1 \leqslant j \leqslant m$, such that 
(a) $\quad \alpha_{i j}-\alpha_{i j}(k) \in_{2 n+m} \mathfrak{m}^{2}, \quad 1 \leqslant i, j \leqslant r$,

(b) $\omega_{i}-\omega_{i}(k) \in{ }_{2 n+m} \mathrm{~m}^{2}, \quad 1 \leqslant i \leqslant n$, and

$$
\gamma_{j}-\gamma_{j}(k) \in_{2 n+m} \mathfrak{m}^{2}, \quad 1 \leqslant j \leqslant m,
$$

(c) $f_{i}(t+\omega(t, x, y), \gamma(t, x, y))=\sum \alpha_{i j}(t, x, y) f_{j}(x, y)$,

$$
1 \leqslant i \leqslant r .
$$

Using (2.19) and (2.21)(b) we see that the map $(\omega(0, x, y), \gamma(0, x, y))$ : $\mathbf{C}_{0}^{n+m} \rightarrow \mathbf{C}_{0}^{n+m}$ is a germ of an isomorphism. But a trivial calculation shows that the value of the jacobian of this map at $0 \in \mathbf{C}^{n+m}$ is the same as the value of the jacobian of the map $(t, \omega(t, x, y), \gamma(t, x, y)): \mathbf{C}_{0}^{2 n+m} \rightarrow \mathbf{C}_{0}^{2 n+m}$ at $0 \in \mathbf{C}^{2 n+m}$. Denoting this later map by $\Omega$, we see that $\Omega=(t, \omega, \gamma): \mathbf{C}_{0}^{2 n+m} \rightarrow$ $\mathbf{C}_{0}^{2 n+m}$ is a germ of an isomorphism.

Let $X_{0}^{\prime} \subset \mathbf{C}_{0}^{2 n+m}$ be the germ of an analytic space defined by the ideal in $2 n+m \Theta$ generated by the germs at $0 \in \mathbf{C}^{2 n+m}$ of $f_{1}(t+x, y), \ldots, f_{r}(t+$ $x, y)$. We have $\mathbf{C}_{0}^{n} \times X_{0} \subset \mathbf{C}_{0}^{2 n+m}$ is defined by the ideal in ${ }_{2 n+m} \theta$ generated by the germs at $0 \in \mathbf{C}^{2 n+m}$ of $f_{1}(x, y), \ldots, f_{r}(x, y)$. By $(2.21)(\mathrm{c})$ we see that $\Omega=(t, \omega, \gamma)$ induces a map $\Omega: \mathbf{C}_{0}^{n} \times X_{0} \rightarrow X_{0}^{\prime}$.

Since $\left\|\alpha_{i j}(k)\right\| \in \mathrm{Gl}\left(r,{ }_{2 n+m} \theta\right),(2.21)(\mathrm{a})$ shows that $\left\|\alpha_{i j}\right\| \in \mathrm{Gl}(r, 2 n+m)$. Using this, and the fact that $\Omega: \mathbf{C}_{0}^{2 n+m} \rightarrow \mathbf{C}_{0}^{2 n+m}$ is an isomorphism, (2.21)(c) actually give $\Omega$ induces an isomorphism $\Omega: \mathbf{C}_{0}^{n} \times X_{0} \rightarrow X_{0}^{\prime}$.

Now the holomorphic vector fields $\partial / \partial t_{1}, \ldots, \partial / \partial t_{n}$ clearly preserve the ideal generated by $f_{1}(x, y), \ldots, f_{r}(x, y)$. Since $\Omega: \mathbf{C}_{0}^{2 n+m} \rightarrow \mathbf{C}_{0}^{2 n+m}$ is an isomorphism we can push these vector fields forward to get germs of holomorphic vector fields $\Omega_{*}\left(\partial / \partial t_{1}\right), \ldots, \Omega_{*}\left(\partial / \partial t_{n}\right)$. Since $\Omega$ induces an isomorphism $\Omega: \mathbf{C}_{0}^{n} \times X_{0} \rightarrow X_{0}^{\prime}$ we see that the $\Omega_{*}\left(\partial / \partial t_{i}\right), 1 \leqslant i \leqslant n$, all preserve the ideal generated by $f_{1}(t+x, y), \ldots, f_{r}(t+x, y)$. Clearly, the holomorphic vector fields $\left(\partial / \partial t_{i}-\partial / \partial x_{i}\right), 1 \leqslant i \leqslant n$, also preserve this ideal. Using (2.21)(b) and (2.19) we easily calculate

$$
\Omega_{*}\left(\frac{\partial}{\partial t_{i}}\right)(0)=\left.\frac{\partial}{\partial t_{i}}\right|_{0}, \quad 1 \leqslant i \leqslant n .
$$

Since $\partial /\left.\partial t_{1}\right|_{0}, \ldots, \partial /\left.\partial t_{n}\right|_{0},\left.\left(\partial / \partial t_{1}-\partial / \partial x_{1}\right)\right|_{0}, \ldots,\left.\left(\partial / \partial t_{n}-\partial / \partial x_{n}\right)\right|_{0}$ are linearly independent we get $\operatorname{dim} C_{1}\left(X_{0}^{\prime}\right) \geqslant 2 n$. Since $\mathbf{C}_{0}^{n} \times X_{0} \cong X_{0}^{\prime}$, Lemma 1.2 gives $\operatorname{dim} C_{1}\left(X_{0}\right) \geqslant n$.

The proof of Theorem 2.1 is now complete. Using it we see Iso $(X, p)_{p}=$ $M(X, p)$. Thus, by Proposition $2.15, \operatorname{dim} \operatorname{Iso}(X, p)_{p}=\operatorname{dim} C_{1}\left(X_{p}\right)$. In particular, we get

REMARK 2.22. $p$ is an isolated point of $\operatorname{Iso}(X, p)$ if and only if $\operatorname{dim} C_{1}\left(X_{p}\right)$ $=0$. 
3. Clustering. Let $V$ be a germ of an analytic space. Recall that by a representative of $V$ one means a pair $(X, p)$ consisting of an analytic space $X$, and a point $p \in X$ such that $V \cong X_{p}$.

Definition 3.1. Let $V$ and $W$ be germs of analytic spaces. I will say that $W$ clusters in $V$ if and only if there is a representative $(X, p)$ for $V$ and a sequence $q_{i} \in X-\{p\}$ such that the $q_{i}$ converge to $p$, and every pair $\left(X, q_{i}\right)$ is a representative for $W$.

Note that if $W$ clusters in $V$ and if $\left(X^{\prime}, p^{\prime}\right)$ is any representative of $V$, then one can find such a sequence $q_{i}^{\prime} \in X^{\prime}-\left\{p^{\prime}\right\}$. Also, clustering is transitive; if $V_{1}$ clusters in $V_{2}$, and $V_{2}$ clusters in $V_{3}$ then $V_{1}$ clusters in $V_{3}$. Finally, if $V$ clusters in $V$ then $\operatorname{dim} C_{1}(V) \geqslant 1$. This last observation follows from Remark 2.22 .

LEMMA 3.2. If $\left\{V_{1}, \ldots, V_{k}\right\}$ is a finite set of germs of analytic spaces with $\operatorname{dim} C_{1}\left(V_{i}\right)=0,1 \leqslant i \leqslant k$, then one can find an $i \in\{1, \ldots, k\}$ such that $V_{i}$ does not cluster in any of the $V_{j}, 1 \leqslant j \leqslant k$.

Proof. If not, we can find a map $\varphi:\{1,2, \ldots, k+1\} \rightarrow\left\{V_{1}, \ldots, V_{k}\right\}$ such that $\varphi(i)$ clusters in $\varphi(i+1)$ for $1 \leqslant i \leqslant k$. But $\varphi$ cannot be injective. Let $i$ and $j, i<j$, be such that $\varphi(i)=\varphi(j)$. Using the transitivity of clustering we get $\varphi(i)$ clusters in itself, so that, by the previous lemma, $\operatorname{dim} C_{1}(\varphi(i)) \geqslant$ 1. This is a contradiction.

We shall not need, but it is interesting to note,

Proposition 3.3. If $V$ and $W$ are germs of analytic spaces, and if $V$ clusters in $W$ and $W$ clusters in $V$, then $V \cong W$ and $\operatorname{dim} C_{1}(V) \geqslant 1$.

Proof. The proof is left to the reader.

4. Decompositions. Throughout this section, all analytic spaces and all germs of analytic spaces will be taken to be reduced. I will use $V, W, V_{1}$, etc. to denote reduced germs of analytic spaces. I will use $X, Y, X_{1}$, etc. to denote reduced analytic spaces. Before proving Theorem 0.3 , I will collect some elementary but useful facts.

If $V=U V_{i}$ is the decomposition of $V$ into irreducible components and $W=\cup W_{j}$ is the decomposition of $W$ into irreducible components, then $V \times W=U\left(V_{i} \times W_{j}\right)$ is the decomposition of $V \times W$ into irreducible components. For a germ $V$ and an integer $d$ we define $N(V, d)$ to be the number of irreducible components of $V$ of dimension $d$, and we define a polynomial $P(V, t)=\Sigma N(V, d) t^{d}$. Then for any $d$ we get $N(V \times W, d)=$ $\Sigma N(V, i) N(W, d-i)$ so that $P(V \times W, t)=P(V, t) P(W, t)$. It follows that if $V_{1} \times W \cong V_{2} \times W$ then $P\left(V_{1}, t\right)=P\left(V_{2}, t\right)$. Finally we have the important observation that if $V \subset W$ and $V \neq W$ then there is a $d$ such that 
$N(V, d)<N(W, d)$. Thus, if $V$ is isomorphic to $W_{1} \subset W$ and $P(V, t)=$ $P(W, t)$ then $W_{1}=W$ and $V \cong W$.

I now prove

THEOREM 0.3. If $V$ is a positive dimensional germ of a reduced analytic space, then $V$ is uniquely decomposable.

Proof. The existence of a decomposition of $V$ into indecomposables is trivial. Any decomposition of maximal length will do. (Note that the length of any decomposition of $V<\operatorname{dim} V$.) Only the uniqueness must be proven. The proof will proceed by induction on $\operatorname{dim} V$.

If $\operatorname{dim} V=1$, then $V$ is indecomposable and there is nothing to prove.

Now suppose $\operatorname{dim} V>1$ and Theorem 0.3 has been proven for all germs of dimension $<\operatorname{dim} V$. We must prove the uniqueness for $V$. I begin with two reductions.

REDUCTION 1. We may assume $V$ is not indecomposable because if $V$ is indecomposable there is nothing to prove.

Now suppose $\left(V_{1}, \ldots, V_{k}\right)$ and $\left(W_{1}, \ldots, W_{l}\right)$ are two decompositions of $V$ with all $V_{i}$ and all $W_{j}$ indecomposable. By Reduction 1 we may assume $k>2$ and $l>2$.

Reduction 2. We may assume $\operatorname{dim} C_{1}(V)=0$.

Proof of Reduction 2. Suppose $\operatorname{dim} C_{1}(V)>0$. Then, by Lemma 1.2, we may reorder the $V_{i}^{\prime}$ 's and the $W_{j}^{\prime}$ 's to achieve $\operatorname{dim} C_{1}\left(V_{1}\right)>0$ and $\operatorname{dim} C_{1}\left(W_{1}\right)$ $>0$. Since $V_{1}$ and $W_{1}$ are indecomposable we get, by applying Lemma 1.3, that $V_{1} \cong W_{1} \cong \mathrm{C}_{0}$. But then, we can use Lemma 1.5 to conclude that $\left(V_{2}, \ldots, V_{k}\right)$ and $\left(W_{2}, \ldots, W_{l}\right)$ give two decompositions into indecomposables of some germ $V^{\prime}$. Since $\operatorname{dim} V^{\prime}=\operatorname{dim} V-1$ the unique decomposability of $V$ follows from the induction hypothesis.

Making use of both reductions (and of Lemma 1.2), let $\left(V_{1}, \ldots, V_{k}\right)$ and $\left(W_{1}, \ldots, W_{l}\right)$ be two decompositions with all $V_{i}$ and $W_{j}$ indecomposable. Then $k \geqslant 2, l \geqslant 2, \operatorname{dim} C_{1}\left(V_{i}\right)=0,1<i<k$, and $\operatorname{dim} C_{1}\left(W_{j}\right)=0,1<j$ $<l$.

Let $n=\max \left\{\operatorname{dim} V_{1}, \ldots, \operatorname{dim} V_{k}, \operatorname{dim} W_{1}, \ldots, \operatorname{dim} W_{l}\right\}$ and let $A=$ $\left\{V_{i} \mid \operatorname{dim} V_{i}=n\right\} \cup\left\{W_{j} \mid \operatorname{dim} W_{j}=n\right\}$. By Lemma 3.2, I can find a $V^{\prime} \in A$ which does not cluster in any element of $A$. Since $V^{\prime}$ clearly cannot cluster in any $W$ with $\operatorname{dim} W<\operatorname{dim} V^{\prime}=n$, we get, in fact, that $V^{\prime}$ does not cluster in any $V_{i}, 1<i<k$, and $V^{\prime}$ does not cluster in any $W_{j}, 1<j<l$.

We may assume that $V^{\prime}$ is isomorphic to the first $r$ of the $V_{i}$ 's and to the first $s$ of the $W_{j}^{\prime}$ 's, and that no other $V_{i}$ or $W_{j}$ is isomorphic to $V^{\prime}$.

We may also assume that $r \geqslant s$. Then $r \geqslant 1$. We set $\gamma=r$ if $r<k$, and $\gamma=k-1$ if $r=k$. Note that $1<\gamma<k-1$.

Let $\left(X_{1}, p(1)\right), \ldots,\left(X_{k}, p(k)\right)$ be representatives for $V_{1}, \ldots, V_{k}$ and let $\left(Y_{1}, q(1)\right), \ldots,\left(Y_{l}, q(l)\right)$ be representatives for $\dot{W}_{1}, \ldots, W_{l}$. 
Shrinking the $Y_{j}$ 's (by replacing each $Y_{j}$ by a small open neighborhood of $\left.q(j) \in Y_{j}\right)$ we may assume that for each $j, 1 \leqslant j \leqslant l$, we have $\operatorname{dim}\left(Y_{j}\right)_{q}<$ $\operatorname{dim} W_{j}$ for every $q \in Y_{j}$. Since $V^{\prime}$ does not cluster in any of the $W_{j}^{\prime}$ s, we may also assume (by further shrinking the $Y_{j}^{\prime}$ 's) that for each $j, 1<j<l$, we have $V^{\prime}$ is not isomorphic to $\left(Y_{j}\right)_{q}$ for any $q \in Y_{j}-\{q(j)\}$.

Since $V_{1} \times \cdots \times V_{k} \cong W_{1} \times \cdots \times W_{l}$, we can find (after shrinking the $X_{i}^{\prime}$ 's) an isomorphism $\psi: X_{1} \times \cdots \times X_{k} \rightarrow U$ (an open neighborhood of $(q(1), \ldots, q(l))$ in $\left.Y_{1} \times \cdots \times Y_{l}\right)$ such that $\psi(p(1), \ldots, p(k))=$ $(q(1), \ldots, q(l))$. This isomorphism will first be used to show $\gamma<s$.

Choose $x(i) \in \operatorname{Reg}\left(X_{i}\right), \gamma+1<i \leqslant k$. Let

$$
\psi((p(1), \ldots, p(\gamma), x(\gamma+1), \ldots, x(k)))=(y(1), \ldots, y(l)) .
$$

Then, using $\left(X_{i}\right)_{p(i)} \cong V_{i}$, we have

$$
\begin{aligned}
V_{1} \times \cdots & \times V_{\gamma} \times\left(X_{\gamma+1}\right)_{x(\gamma+1)} \times \cdots \times\left(X_{k}\right)_{x(k)} \\
& \simeq\left(Y_{1}\right)_{y(1)} \times \cdots \times\left(Y_{l}\right)_{y(l)} .
\end{aligned}
$$

Let $h=\operatorname{dim}\left(X_{\gamma+1}\right)_{x(\gamma+1)}+\cdots+\operatorname{dim}\left(X_{k}\right)_{x(k)}$. For each $j, 1<j<l$, let $m(j)=\operatorname{dim} C_{1}\left(\left(Y_{j}\right)_{y(j)}\right)$. Then we can write $\left(Y_{j}\right)_{y(j)}=W_{j}^{\prime} \times \mathbf{C}_{0}^{m(j)}$ where $\operatorname{dim} C_{1}\left(W_{j}^{\prime}\right)=0$. Setting $m=\Sigma m(j),(4.1)$ becomes

$$
V_{1} \times \cdots \times V_{\gamma} \times \mathbf{C}_{0}^{h} \cong W_{1}^{\prime} \times \cdots \times W_{l}^{\prime} \times \mathbf{C}_{0}^{m} .
$$

Using Lemma 1.2 we get $h=m$. Using Lemma 1.5 repeatedly we get

$$
V_{1} \times \cdots \times V_{\gamma} \cong W_{1}^{\prime} \times \cdots \times W_{l}^{\prime} \text {. }
$$

By the construction of the $Y_{j}^{\prime}$ 's and our choice of $V^{\prime}$ we get

$$
\operatorname{dim} V^{\prime} \geqslant \operatorname{dim} W_{j} \geqslant \operatorname{dim}\left(\left(Y_{j}\right)_{y(j)}\right) \geqslant \operatorname{dim} W_{j}^{\prime}, \quad 1 \leqslant j \leqslant l .
$$

It is worth noting that $\operatorname{dim} V^{\prime}=\operatorname{dim} W_{j}^{\prime}$ only if $\left(Y_{j}\right)_{y(j)} \cong W_{j}^{\prime}$.

Since $V_{1} \cong \cdots \cong V_{\gamma} \cong V^{\prime}$, we get from (4.4),

$$
\gamma\left(\operatorname{dim} V^{\prime}\right)=\sum \operatorname{dim} W_{j}^{\prime}
$$

Let $L=\left\{j \mid 1<j<l\right.$ and $\left.\operatorname{dim} W_{j}^{\prime}>0\right\}$. From (4.4) and (4.5) we see that $L$ contains at least $\gamma$ integers.

On the other hand, $\operatorname{dim} V>\operatorname{dim}\left(V_{1} \times \cdots \times V_{\gamma}\right)$, so by our induction hypothesis $V_{1} \times \cdots \times V_{\gamma}$ is uniquely decomposable. Since each $V_{i}$ is indecomposable, it follows that no decompositions of $V_{1} \times \cdots \times V_{\gamma}$ can have length greater than $\gamma$. This shows that $L$ contains at most $\gamma$ integers. Thus, $L$ contains precisely $\gamma$ integers, and the $W_{j}^{\prime}, j \in L$, give the terms of a decomposition of $V_{1} \times \cdots \times V_{\gamma}$ and all the $W_{j}^{\prime}, j \in L$, are indecomposable. Using the fact that $V_{1} \times \cdots \times V_{\gamma}$ is uniquely decomposable, and using $V_{i} \cong V^{\prime}$ for $1<i<\gamma$, we see

$$
V^{\prime} \cong W_{j}^{\prime} \text { for all } j \in L
$$


Thus $\operatorname{dim} V^{\prime}=\operatorname{dim} W_{j}^{\prime}$ for $j \in L$. But as noted above, this gives $\left(Y_{j}\right)_{y(j)} \cong$ $W_{j}^{\prime}$ and thus $\left(Y_{j}\right)_{y(j)} \cong V^{\prime}$ for $j \in L$. By our construction of the $Y_{j}^{\prime}$ 's, we see that, for $j \in L, y(j) \notin Y_{j}-\{q(j)\}$ so that $y(j)=q(j)$. Thus for $j \in L$ we get $V^{\prime} \cong\left(Y_{j}\right)_{q(j)} \cong W_{j}$. This gives $s \geqslant \gamma=\operatorname{Card}(L)$, and $L \subset\{j \mid 1 \leqslant j \leqslant s\}$.

The proof of the theorem is now reduced to two cases.

Case 1. $s=\gamma$. In this case $L=\{j \mid 1 \leqslant j \leqslant s\}$. Then, what we have just seen is that for $x(i) \in \operatorname{Reg}\left(X_{i}\right), s+1 \leqslant i \leqslant k$,

$$
\begin{aligned}
\psi((p(1), \ldots, p(s), x(s+1), \ldots, x(k))) \\
\quad=(q(1), \ldots, q(s), y(s+1), \ldots, y(l)) .
\end{aligned}
$$

Since $\operatorname{Reg}\left(X_{s+1}\right) \times \cdots \times \operatorname{Reg}\left(X_{k}\right)$ is dense in $X_{s+1} \times \cdots \times X_{k}$, it follows that $\psi\left(\{(p(1), \ldots, p(s))\} \times X_{s+1} \times \cdots \times X_{k}\right)$ is contained in $\{(q(1), \ldots, q(s))\} \times Y_{s+1} \times \cdots \times Y_{l}$.

In other words, we have just established

FACT 4.7. $V_{s+1} \times \cdots \times V_{k}$ is isomorphic to some $W^{\prime} \subset W_{s+1} \times \cdots \times$ $W_{l}$.

Since $V_{i} \cong W_{i} \cong V^{\prime}$ for $1 \leqslant i \leqslant s$ we get $V_{1} \times \cdots \times V_{s} \cong W_{1}$ $\times \cdots \times W_{s}$. We also have

$$
\begin{aligned}
V & \cong\left(V_{1} \times \cdots \times V_{s}\right) \times\left(V_{s+1} \times \cdots \times V_{k}\right) \\
& \cong\left(W_{1} \times \cdots \times W_{s}\right) \times\left(W_{s+1} \times \cdots \times W_{l}\right) .
\end{aligned}
$$

But then, the introductory remarks to this section show that we have established

FACT 4.8. $P\left(V_{s+1} \times \cdots \times V_{k}, t\right)=P\left(W_{s+1} \times \cdots \times W_{l}, t\right)$.

Again applying those introductory remarks we may conclude $V_{s+1}$ $\times \cdots \times V_{k} \cong W_{s+1} \times \cdots \times W_{l}$. Since $\operatorname{dim} V>\operatorname{dim}\left(V_{s+1} \times \cdots \times V_{k}\right)$ we may apply the induction hypothesis to conclude $k-s=l-s$ (so that $k=l$ ), and (after permuting the $W_{s+1}, \ldots, W_{k}$ ) we have $V_{i} \cong W_{i}$ for $s+1 \leqslant i \leqslant k$. We already had $V_{i} \cong W_{i} \cong V^{\prime}$ for $1 \leqslant i \leqslant s$. This completes the proof of the theorem in Case 1 .

Case 2. $s>\gamma$. In this case $r>\gamma$. But, by the definition of $\gamma$ we see that this implies $r=k$ and $\gamma=k-1$. But then we also have $s=k$.

In this case we have $V=V_{1} \times \cdots \times V_{s}$ with all $V_{i} \cong V^{\prime}$. Then $\operatorname{dim} V=$ $s\left(\operatorname{dim} V^{\prime}\right)$. Since $\Sigma \operatorname{dim} W_{j}=s\left(\operatorname{dim} V^{\prime}\right)$, and since $s$ of the $W_{j}$ are isomorphic to $V^{\prime}$, we see that there are no $W_{j}$ 's except those isomorphic to $V^{\prime}$. Thus $l=s$ so that $k=l$, and for each $i, 1 \leqslant i \leqslant k, V_{i} \cong W_{i} \cong V^{\prime}$.

This completes the induction step and the proof.

\section{REFERENCES}

1. J. Becker and R. Ephraim, Real and complex products of singularities (in preparation).

2. R. Ephraim, The Cartesian product structure and $C^{\infty}$ equivalences of singularities, Trans. Amer. Math. Soc. 224 (1976), 299-311. 
3. G. Fischer, Complex analytic geometry, Lecture Notes in Math., vol. 538, Springer-Verlag, Berlin and New York, 1976.

4. D. Mumford, Introduction to algebraic geometry (preliminary version of the first three chapters), Lecture notes, Harvard Univ., Cambridge, Mass.

5. A. Seidenberg, Analytic products, Amer. J. Math. 91 (1969), 577-590.

6. On analytically equivalent ideals, Inst. Hautes Etude Sci. Publ. Math. No. 36 (1969), 69-74.

7. J. Wavrik, $A$ theorem on solutions of analytic equations with applications to deformations of complex structures, Math. Ann. 216 (1975), 127-142.

8. H. Whitney, Local properties of analytic varieties, Differential and Combinatorial Topology, Princeton Univ. Press, Princeton, N. J., 1965.

9. , Complex analytic varieties, Addison-Wesley, Reading, Mass., 1972.

Department of Mathematics, CUNY, Lehman College, New York, New York 10468 